\title{
CLINICAL PRACTICE Clinical Vignettes \\ That Which Bends Up: A Case Report and Literature Review of Chikungunya Virus
}

\author{
Shana M. Peper, MD', Benjamin J. Monson, MD', Trevor Van Schooneveld, MD², \\ and Christopher J. Smith, MD \\ 'Division of Education, Department of Internal Medicine, University of Nebraska Medical Center, Omaha, NE, USA; ${ }^{2}$ Division of Infectious Disease, \\ Department of Internal Medicine, University of Nebraska Medical Center, Omaha, NE, USA; ${ }^{3}$ Division of General Internal Medicine, Department of \\ Internal Medicine, University of Nebraska Medical Center, Omaha, NE, USA.
}

We present a case of chikungunya virus (CHIKV) in a 39year-old female who developed an acute febrile illness marked by polyarthralgia and rash after returning from Saint Lucia. This epidemic-prone pathogen is increasingly likely to be encountered by primary care and hospital physicians in the coming months. The virus was first locally transmitted in the Caribbean in December 2013 and has since spread to 44 countries and 47 US states, affecting a suspected 1.2 million people. A mosquitoborne virus, CHIKV causes a severe and symmetric polyarthralgia that can relapse for months to years, creating debilitating illness and profound socioeconomic consequences. Current treatment is limited to supportive measures, which are dependent on nonsteroidal antiinflammatory drugs. Research into immunomodulatory agents, antiviral therapies, and vaccines is ongoing. Prevention remains key in slowing the spread of disease. Patient education should focus on personal protective measures, such as insect repellant and remaining indoors, while public health departments should implement strategies to control vector breeding grounds. Given the possibility of relapsing and debilitating disease, general internists should consider CHIKV in the differential diagnosis of a returning traveler with acute onset of fever, polyarthralgia, and rash.

KEY WORDS: chikungunya; symmetric polyarthralgia; mosquitotransmitted virus; Caribbean; febrile rash.

J Gen Intern Med 31(5):576-81

DOI: $10.1007 / \mathrm{s} 11606-015-3459-3$

(c) Society of General Internal Medicine 2015

\section{CASE REPORT}

A 39-year-old African-American female presented to a Midwestern emergency department with 3 days of fever, pruritic rash, and diffuse arthralgias. She complained of severe pain in the small joints of her hands, wrists, hips, knees, and ankles. Accompanying symptoms included generalized myalgia, headache, photophobia, and diarrhea. She denied oral ulcers,

Presented in abstract form at the Society of General Internal Medicine 38th Annual Meeting, April 22-25, 2015.

Received February 4, 2015

Revised May 20, 2015

Accepted June 18, 2015

Published online July 21, 2015 serositis, hair loss, and easy bruising or bleeding. Her past medical history was significant for hypothyroidism, but she was not taking any medications. Family history was noncontributory. She worked as a nurse and denied any needlestick injuries or high-risk sexual behaviors. She was a former smoker without alcohol or recreational drug use. She had returned 1 week ago from Saint Lucia and recalled multiple insect bites, but no other exposures.

The patient's vital signs were within normal limits. She demonstrated mild photophobia, but lacked meningismus. She had tender anterior cervical lymphadenopathy. Her bilateral distal interphalangeal, proximal interphalangeal, metacarpal, wrist, knee, and ankle joints were tender to palpation, but did not exhibit synovitis. Active range of motion (ROM) was reduced in these joints due to pain, but passive ROM was normal. There was a faintly erythematous, maculopapular eruption over the anterior surface of both shins and circumferentially around the forearms, sparing the palms and soles. The remainder of her examination was normal.

At this point, the differential diagnosis of the patient's presentation was divided into infectious versus rheumatologic conditions. Given her recent travel and a history of insect bites, an arthropod-borne illness, such as chikungunya virus (CHIKV) or dengue virus (DENV), seemed most likely. Other infectious possibilities, considered less likely based on her history and exam, included acute human immunodeficiency virus (HIV), leptospirosis, acute mononucleosis, and acute rheumatic fever. The absence of synovitis and short duration of symptoms made rheumatologic diagnoses, such as rheumatoid arthritis (RA), systemic lupus erythematous (SLE), and post-infectious arthritis, less likely.

Laboratory tests revealed a normal complete blood count with differential, metabolic panel, liver function tests, partial thromboplastin time, international normalized ratio, thyroid stimulating hormone, and urinalysis. Chest x-ray and electrocardiogram were unremarkable. Lumbar puncture was withheld due to lack of meningismus and systemic toxicity. HIV testing was not performed as the patient lacked risk factors.

After consultation with the infectious disease service, the patient was presumptively diagnosed with $\mathrm{CHIKV}$, due to her severe symmetric polyarthralgia and lack of coagulopathy, neutropenia, and thrombocytopenia, which are more common 


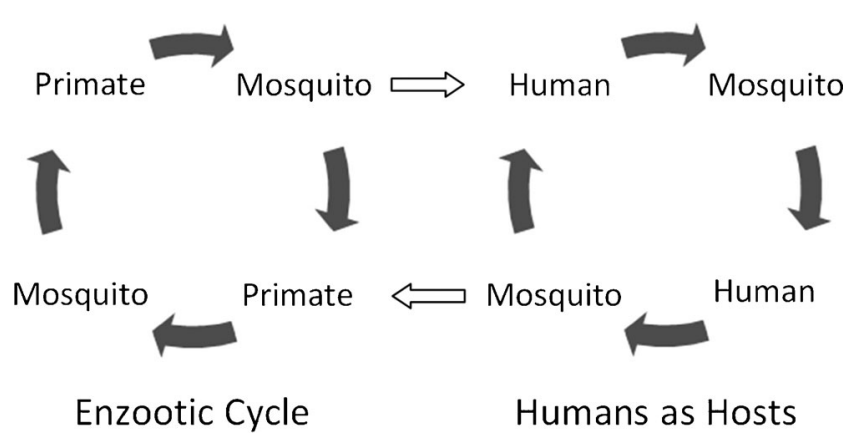

Figure 1. CHIKV is endemic in nonhuman primates, where it exists in an enzootic cycle between the animal and the Aedes mosquito. However, the virus can be transmitted to a human host via the mosquito and become established in a human-mosquito cycle without the need for an animal reservoir.

with DENV. This was further supported by her recent travel to an endemic region, where debilitating polyarthralgia has a positive predictive value of $80 \%$ for chikungunya viremia. ${ }^{1}$

The patient received supportive care with intravenous volume replacement and analgesics with rapid resolution of fever, rash, photophobia, myalgia, and diarrhea. Despite scheduled ibuprofen and acetaminophen, her severe polyarthralgia persisted. On hospital day 4, she was treated with methylprednisolone $40 \mathrm{mg}$ with subsequent improvement in her pain. She was discharged on hospital day 6 with ibuprofen, acetaminophen, and an oral prednisone taper. After discharge, CHIKV IgM enzyme-linked immunosorbent assay (ELISA) testing returned positive; DENV testing was negative.

\section{EPIDEMIOLOGY}

Borrowed from the Kimakonde word for "that which bends up," chikungunya is a RNA alpha virus of the Togaviridae family and is transmitted by the aggressive Aedes aegypti and Aedes albopictus mosquitoes. ${ }^{2}$ Although the virus is endemic in nonhuman primates, ${ }^{3}$ it can be transferred from human to mosquito without an animal reservoir (Fig. 1). ${ }^{4}$ Humans serve as the primary amplifying host, as high levels of viremia during acute illness allow for infection of biting mosquitoes. ${ }^{5}$

CHIKV was first isolated in Tanzania in 1953, but spread to Thailand by $1958 .^{3}$ A 2004 outbreak in Kenya sparked transmission to Mozambique, India, Sri Lanka, and Southeast Asia. ${ }^{4}$ By the end of 2005, 1.3 million cases were suspected in India after 32 years of absent disease activity. ${ }^{3}$ Attack rates as high as $75 \%$ were reported in some locations, such as $\mathrm{La}$ Réunion and Lamu Island. ${ }^{3,6}$ During this same outbreak, local transmission was observed for the first time in Europe, the Middle East, and Papua New Guinea. ${ }^{3}$

CHIKV reached the Western Hemisphere in December 2013 with the first local transmission of the virus on the Caribbean island of St. Martin. ${ }^{3}$ The virus has since spread to 44 countries and territories, including the United States, and has affected 1.2 million people (Fig. 2). ${ }^{7}$ As of February 2015, 47 states have reported a total of 2492 cases (Fig. 3). ${ }^{8}$ Florida accounts for the largest number of infections, including all 11 known cases of locallytransmitted CHIKV. ${ }^{9}$

The presence of Ae. aegypti and Ae. albopictus throughout the subtropical and temperate zones of the US suggests that the spread of CHIKV into new geographic locations is probable (Figs. 4 and 5). ${ }^{3,6}$ While the CHIK $\mathrm{V}$ strain circulating in the Caribbean is limited in its ability to use Ae. albopictus as a vector, ${ }^{3}$ there is the possibility of the virus establishing itself in a mosquitohuman or mosquito-vertebra cycle in the US or other parts of the Western Hemisphere. ${ }^{11}$

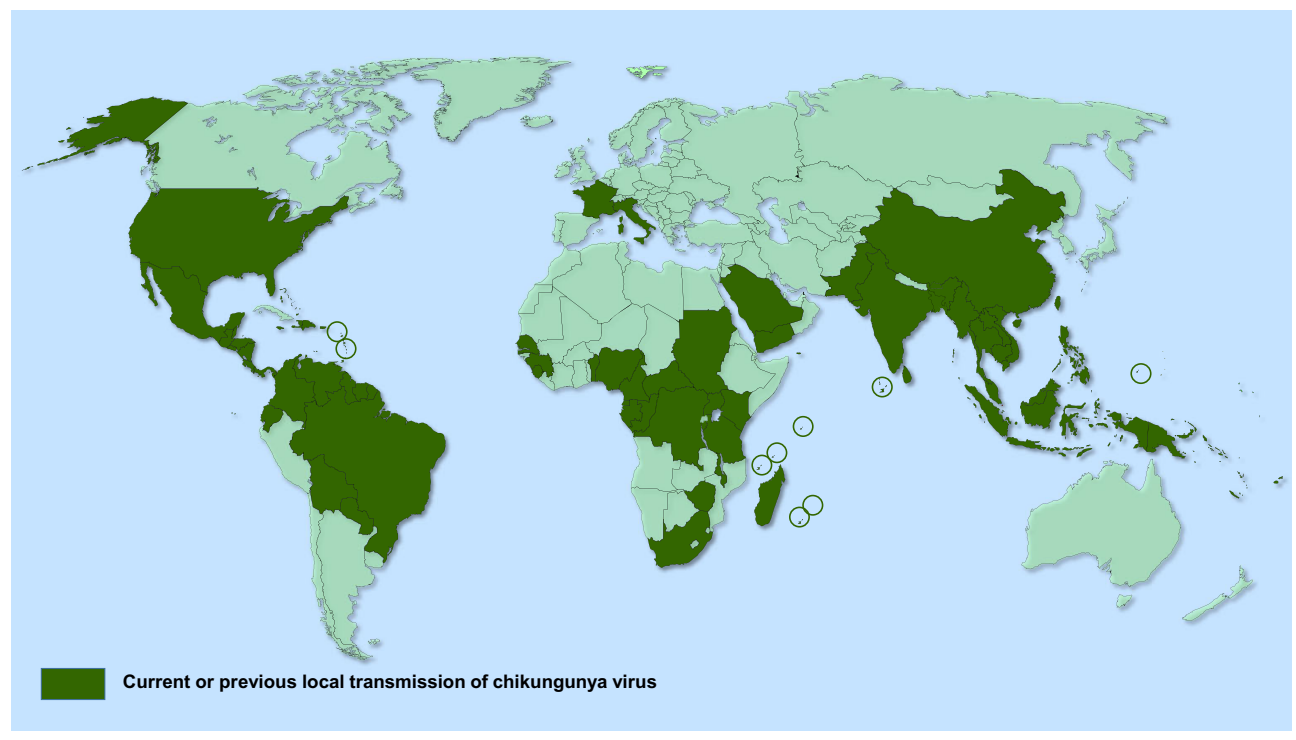

Figure 2. Countries with local transmission of CHIKV, as of March 2015 . $^{7}$ 


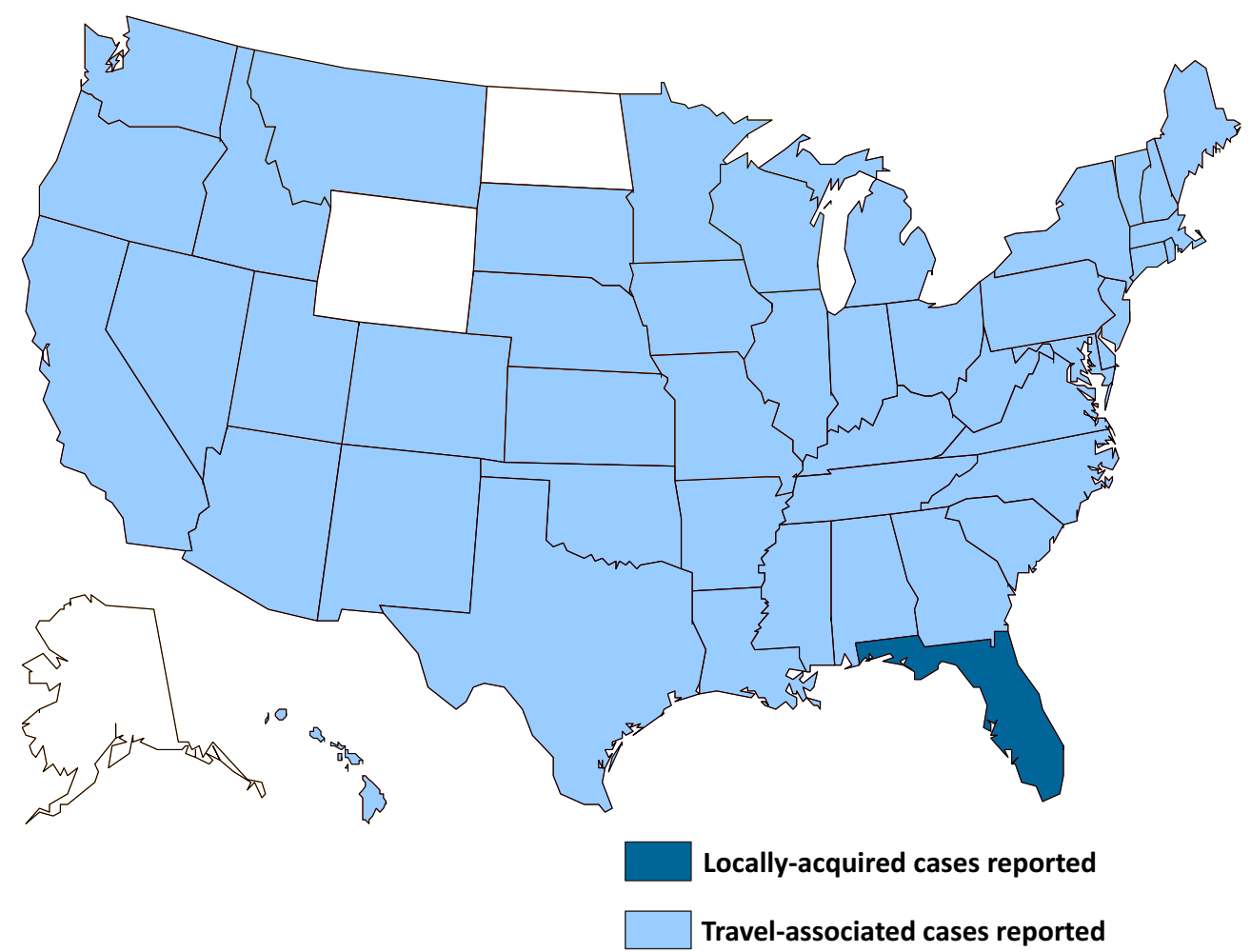

Figure 3. States with confirmed cases of CHIKV infection, as of February 2015. ${ }^{8}$

\section{CLINICAL MANIFESTATIONS, DIFFERENTIAL DIAG NOSIS, AND DIAGNOSIS}

CHIKV infection is marked by acute onset of high fever and symmetric polyarthralgia, primarily of the small joints. ${ }^{2}$ Fifty percent of patients will experience a non-specific maculopapular, pruritic eruption on the trunk and extremities. ${ }^{2}$ Other symptoms include headache, myalgia, and vomiting. ${ }^{5}$ Examination may reveal arthritis, tenosynovitis, lymphadenopathy, conjunctivitis, uveitis, and erythema of the external ear, suggestive of chondritis. ${ }^{1}$ The most common laboratory abnormality is lymphopenia, the severity of which correlates with the degree of viremia. ${ }^{1}$ Less frequently, thrombocytopenia, elevated transaminases, and hypocalcemia may occur in

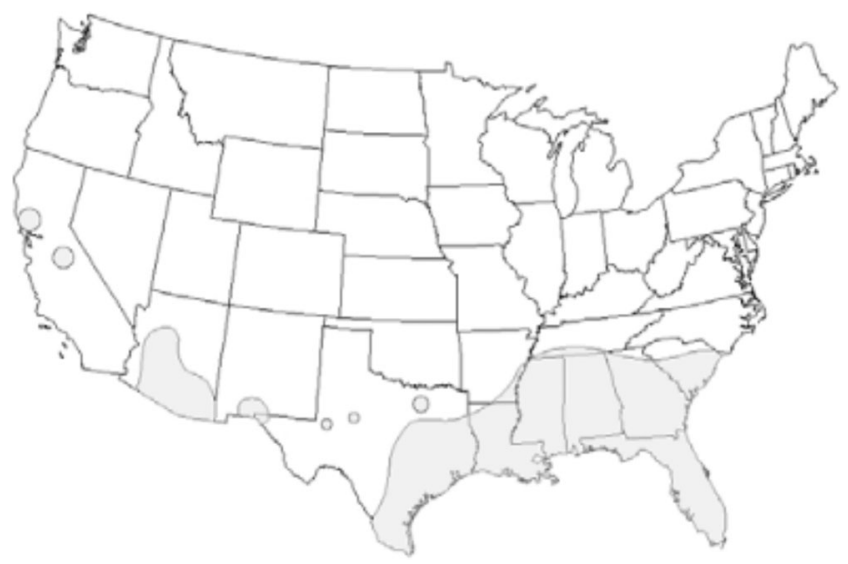

Figure 4. Distribution of Ae. aegypti in the US. ${ }^{10}$ the acute infection. ${ }^{1}$ Coagulopathy and hemorrhagic complications are exceedingly rare with CHIKV and should prompt evaluation for DENV. ${ }^{1}$

CHIKV has an incubation period of 2-12 days, and the subsequent viremia lasts 4-6 days. ${ }^{2,12}$ The duration of viremia correlates both with acute illness and infectivity. ${ }^{12}$ Acute symptoms typically resolve within $1-2$ weeks, ${ }^{4}$ but up to $60 \%$ of patients may experience relapsing and disabling arthralgias and fatigue for months to years after the acute infection subsides. ${ }^{4,5}$ CHIKV has a mortality rate of less than $1 \%$, but vulnerable patients, such as the elderly, are at higher risk for life-threatening disease, including meningoencephalitis, myocarditis, hepatitis, or nephritis. ${ }^{5}$

DENV, also transmitted by Aedes mosquito in the same endemic locations, is a common mimicker of CHIKV as it also causes fever and polyarthralgia. Differentiating the two viral infections can prove difficult. CHIKV is more likely to cause a severe and persistent arthralgia/arthritis, rash, and lymphopenia, whereas DENV more frequently presents with neutropenia and thrombocytopenia, and may progress to severe complications, such as hemorrhage and dengue shock syndrome (Fig. 6). ${ }^{5}$ It is possible for patients to be co-infected with both viruses since they share the same vector. ${ }^{2,4,13} \mathrm{~Pa}$ tients suspected of either CHIKV or DENV should be tested for both viruses to rule-out coinfection. ${ }^{2}$

Also included in the differential of CHIKV are leptospirosis, malaria, yellow fever, influenza, rickettsia, rubella, parvovirus, adenovirus, post-infectious arthritis, HIV, and rheumatologic conditions. ${ }^{5,11,14}$ Acute CHIKV infection is suggested by 


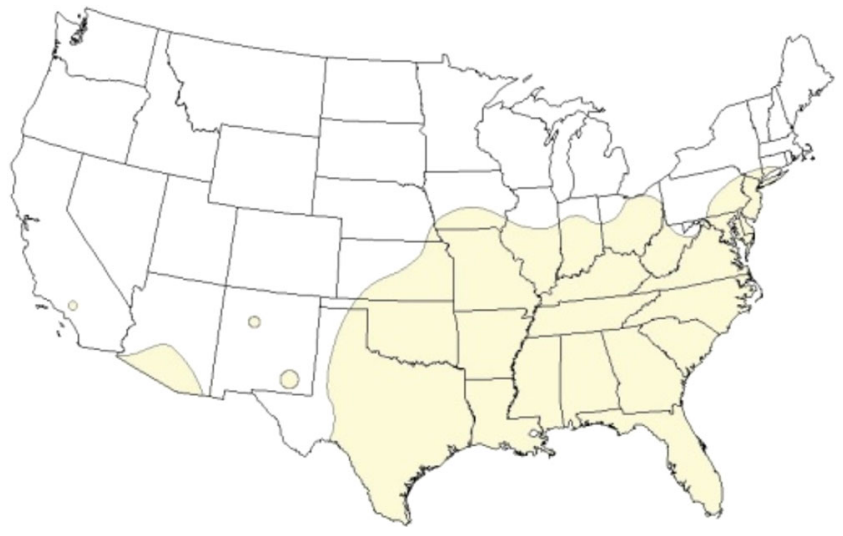

Figure 5. Distribution of Ae. albopictus in the US. ${ }^{10}$

recent travel to an endemic area followed by acute onset of severe symmetric polyarthralgia. ${ }^{1}$ It should be noted that chronic arthralgias from CHIKV infection can mimic RA and provide a diagnostic dilemma. In a recent study, eight of ten patients with chronic symmetric polyarthritis due to CHIK $\mathrm{V}$ also met criteria for RA based on the 2010 American College of Rheumatology/European League Against Rheumatism criteria, highlighting the overlapping clinical features of the two diseases. ${ }^{15}$ Although three of the eight patients had positive antinuclear antibodies, none had rheumatoid factor or anti-cyclic citrullinated protein antibodies. ${ }^{15}$ The acute onset (within hours) of widespread severe arthralgias in CHIKV can differentiate it from RA, underscoring the importance of history in the diagnosis. ${ }^{15}$

The number of cases of CHIKV may actually be underestimated due to the difficulty of diagnosis. The gold standard remains virus isolation, but this is seldom used. ${ }^{13}$ During the acute phase of illness (days 1-8 from symptom onset), diagnosis can be made with reverse transcription polymerase chain reaction (RT-PCR) or IgM serology, although
IgM levels may not rise until 5-7 days after the onset of symptoms. ${ }^{5}$ In the convalescent phase (2-3 weeks after symptoms), IgG may be used to confirm the diagnosis. ${ }^{2}$ It is recommended that both RT-PCR and serology be used to diagnose $\mathrm{CHIKV}$, as false negative results are common with one test alone. ${ }^{16}$ For patients with CHIKV meningoencephalitis, cerebrospinal fluid (CSF) typically demonstrates elevated protein levels and a pleocytosis $(<100$ cells $/ \mu l)$ with a predominance of monocytes. ${ }^{17}$ CHIKV RNA is not routinely detected in CSF. ${ }^{17}$ Several laboratories in the US, including the Centers for Disease Control and Prevention (CDC), perform serology and RT-PCR testing for CHIKV, and results take 4-14 days upon receipt of the sample. ${ }^{18}$ The CDC recommends contacting state health departments for information regarding diagnostic testing.

\section{TREATMENT}

There are no evidence-based treatment recommendations for CHIKV infection, and treatment is largely supportive with nonsteroidal anti-inflammatory drugs (NSAIDs). ${ }^{19}$ For patients with refractory or relapsing symptoms, glucocorticoids and physical therapy have shown some benefit. ${ }^{2,20}$ Duration of treatment is individualized to the duration of symptoms.

Current therapeutic research is targeted at the development of antivirals, monoclonal antibodies, and immunomodulatory drugs. ${ }^{3}$ Convalescent serum from patients with previous CHIKV is able to both prevent and modify the course of the illness in mice, and a mouse monoclonal antibody has been shown to prevent viral release from cells. ${ }^{21}$ Treatment of chronic arthralgias is challenging with the use of traditional rheumatologic agents, such as methotrexate, sulfasalazine, and

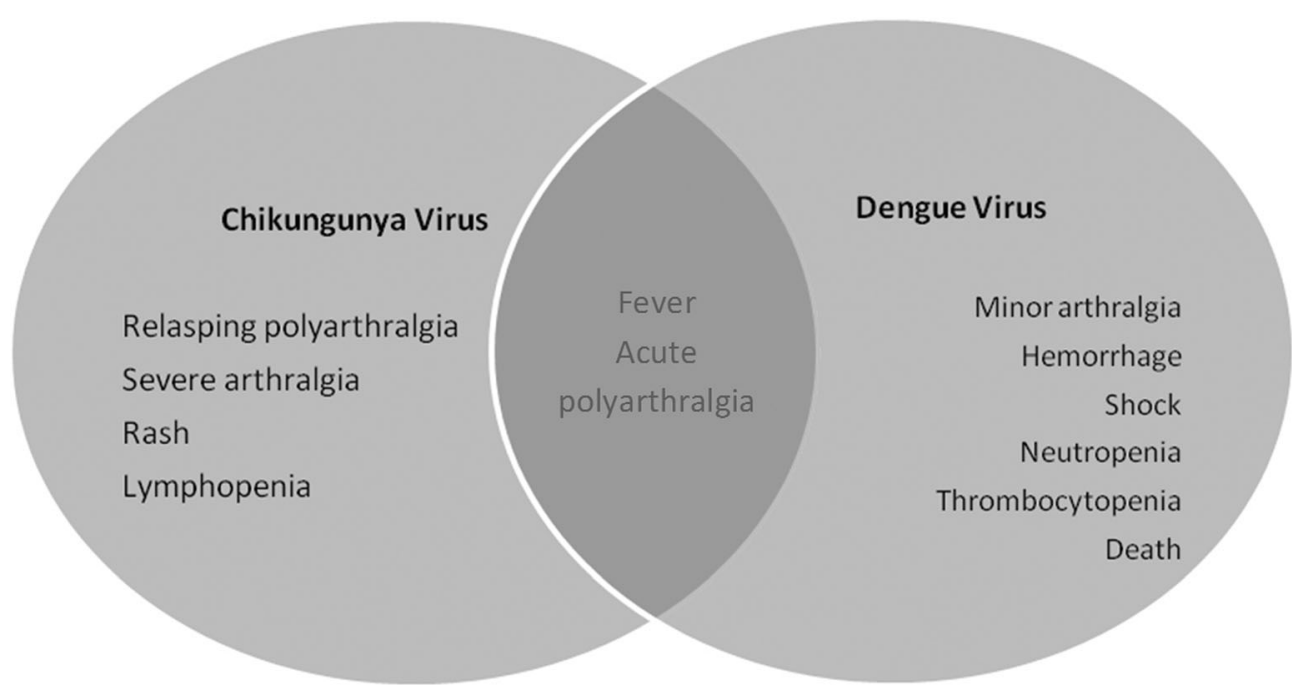

Figure 6. Although both chikungunya and dengue present with fever and acute polyarthralgia, there are important clinical signs to help distinguish between the two viruses. 
chloroquine, demonstrating mixed results in both animal models and small clinical trials. ${ }^{20}$

\section{IMPACT AND PREVENTION}

Global travel facilitates the spread of CHIKV, and $52 \%$ of travelers to the Caribbean have the US as their final destination. ${ }^{6}$ Heavily populated urban centers with little immunity, coupled with the broad distribution of the mosquito vector and the high viral titers of returning infected travelers, suggest that the US is at high risk for an epidemic. $^{22}$ It is estimated that over 500 million people in the Americas are at risk of CHIKV infection, given the distribution of the virus and its vector. ${ }^{23}$ Additionally, the impact of CHIKV outbreaks should not be underestimated as infections can quickly overwhelm health care systems and significantly impact local economies. During the $\mathrm{La}$ Réunion epidemic, more than 47,000 cases were diagnosed in 1 week, with the resultant loss of the ablebodied workforce requiring emergency economic rescue with crisis funding. ${ }^{22}$

Disease prevention is the most effective method for controlling the spread of CHIKV. The Aedes species responsible for viral spread are aggressive mosquitoes, highlighting the need for protective clothing and insect repellent. ${ }^{3}$ Keeping windows and doors closed is also protective. Patients diagnosed with CHIKV should remain indoors during the first week of acute illness, as biting mosquitoes are easily infected when high levels of viremia are present. $^{4,24}$ Surveillance of cases, spraying for vectors, and eliminating vector breeding-sites, such as standing water, are worthwhile efforts to limit distribution of virus and vector. $^{22}$

Although no vaccine yet exists, efforts have been ongoing since the 2004 Kenyan outbreak. Antibodies derived from natural infection are believed to provide protection against future infection, and humoral immunity has been shown to protect against viremia in animal models. ${ }^{25}$ A virus-like particle (VLP) vaccine candidate has been shown to protect nonhuman primates from infection and recently completed Phase I clinical trials. ${ }^{26}$ Antibody development elicited by the vaccine lasted for 6 months, and concentrations at 4 weeks were similar to antibody concentrations in those with natural infection. ${ }^{26}$ Safety data suggests that the vaccine is well-tolerated, but further research is necessary to confirm clinical efficacy. ${ }^{26}$

\section{CONCLUSION}

CHIKV is a global pathogen with potentially devastating economic consequences that has recently spread into the Western Hemisphere, including the US. It is crucial that the diagnosis of CHIKV be considered in patients with fever, polyarthralgia, and recent travel to endemic areas, such as the Caribbean, Central and South America, and Asia. Current treatment options are limited to supportive measures, but promising research into a vaccine is ongoing. Coordinated, prompt public health efforts will be essential in limiting the spread of CHIKV and mitigating the impact of outbreaks.

Acknowledgements: Permission was received from the CDC for use and adaptation of the referenced maps.

Funding: There were no funding sources for this study.

Conflicts of Interest: The authors declare that they do not have a conflict of interest.

Corresponding Author: Shana M. Peper, MD; Division of Education, Department of Internal Medicine, University of Nebraska Medical Center, 982055 Nebraska Medical Center, Omaha, NE 68198-2055, USA (e-mail: shana.peper@unmc.edu).

\section{REFERENCES}

1. Weaver SC, Lecuit M. Chikungunya virus and the global spread of a mosquito-borne disease. N Engl J Med. 2015;372:1231-1239.

2. Hamer DH, Chen LH. Chikungunya: establishing a new home in the Western Hemisphere. Ann Intern Med. 2014;161:827-828.

3. Morrison TE. Reemergence of chikungunya virus. J Virol. 2014;88:1164411647.

4. Stamm LV. Chikungunya: emerging threat to the United States. JAMA Dermatol. 2014. doi:10.1001/jamadermatol.2014.2034.

5. Staples JE, Fischer M. Chikungunya virus in the Americas-what a vectorborne pathogen can do. N Engl J Med. 2014;371:887-889.

6. Mowatt L, Jackson ST. Chikungunya in the Caribbean: an epidemic in the making. Infect Dis Ther. 2014;3:63-68.

7. Centers for Disease Control and Prevention. Chikungunya virus: geographical distribution. Available at: http://www.cdc.gov/chikungunya/ geo/index.html. Accessed 16 June 2015.

8. Centers for Disease Control and Prevention. Chikungunya virus: 2014 provisional data for the United States. Available at: http://www.cdc.gov/ chikungunya/geo/united-states-2014.html. Accessed June 16, 2015.

9. Kendrick K, Stanek D, Blackmore C. Notes from the field: transmission of chikungunya virus in the continental United States-Florida, 2014. MMWR Morb Mortal Wkly Rep. 2014;63(48):1137.

10. Centers for Disease Control and Prevention. Chikungunya: information for vector control programs. Available at: http://www.cdc.gov/chikungunya/ pdfs/CHIKV_VectorControl.pdf. Accessed 16 June 2015.

11. Morens DM, Fauci AS. Chikungunya at the door-déjà vu all over again? N Engl J Med. 2014;371:885-887.

12. Lindsey NP, Prince HE, Kosoy O, et al. Chikungunya virus infections among travelers-United States, 2010-2013. Am J Trop Med Hyg. 2015;92(1):82-87.

13. Cecilia D, Kakade M, Alagarasu K, et al. Development of a multiplex real-time RT-PCR assay for simultaneous detection of dengue and chikungunya viruses. Arch Virol. 2014. doi:10.1007/s00705-014-2217-x.

14. Weaver SC. Arrival of chikungunya virus in the new world: prospects for spread and impact on public health. PLoS Negl Trop Dis. 2014;8:e2921. doi:10.1371/journal.pntd.0002921.

15. Miner JJ, Aw Yeang HX, Fox JM, et al. Brief report: chikungunya viral arthritis in the United States: a mimic of seronegative rheumatoid arthritis. Arthritis Rheum. 2015;67(5): 1214-1220.

16. Kuehn BM. Chikungunya virus transmission found in the United States: US health authorities brace for wider spread. JAMA. 2014;312:776-777.

17. Nelson J, Waggoner JJ, Sahoo MK, Grant PM, Pinsky BA. Encephalitis caused by chikungunya virus in a traveler from the kingdom of Tonga. $J$ Clin Microbiol. 2014;52(9):3459-61.

18. Centers for Disease Control and Prevention. Chikungunya virus: diagnostic testing. Available at: http://www.cdc.gov/chikungunya/hc/diagnostic. html. Accessed 16 June 2015. 
19. Javelle E, Ribera A, Degasne I, Gauzere BA, Marimoutou C, Simon F. Specific management of post-chikungunya rheumatic disorders: a retrospective study of 159 cases in Reunion Island from 2006-2012. PLoS Negl Trop Dis. 2015;9(3):e0003603.

20. Parashar D, Cherian S. Antiviral perspectives for chikungunya virus. Biomed Res Int. 2014. doi:10.1155/2014/631642.

21. Masrinoul P, Puiprom O, Tanaka A, et al. Monoclonal antibody targeting chikungunya virus envelope 1 protein inhibits virus release. Virology. 2014;464-465:111-117.

22. Roth A, Hoy D, Horwood PF, et al. Preparedness for threat of chikungunya in the Pacific. Emerg Infect Dis. 2014;20:e130696. doi:10. 3201/eid2008.130696.
23. Powers AM. Risks to the Americas associated with the continued expansion of chikungunya virus. J Gen Virol. 2014. doi:10.1099/vir.0.070136-0.

24. Centers for Disease Control and Prevention. Chikungunya virus: clinical evaluation and disease. Available at: http://www.cdc.gov/chikungunya/ hc/clinicalevaluation.html. Accessed 16 June 2015.

25. Sheela PJ, Sumathy K. Serological correlates of immune protection conferred by chikungunya virus infection. Acta Virol. 2013;57(4):471-473.

26. Powers AM. Chikungunya virus control: is a vaccine on the horizon? Lancet. 2014;384:2008-2009. 